\title{
THE FIRST BEAM ACCELERATION TEST USING HIGH GRADIENT CAVITY AT HIMAC
}

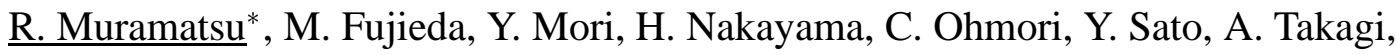 \\ T. Uesugi, M. Yamamoto, M. Yoshii, KEK-Tanashi, Tokyo 188-8501, Japan \\ M. Kanazawa, K. Noda, NIRS, Chiba 263-8555, Japan
}

\section{Abstract}

The first beam acceleration test using a High Gradient Cavity (HGC) loaded with Magnetic Alloy (MA) cores has been carried out at the HIMAC (Heavy Ion Medical Accelerator in Chiba). Acceleration of heavy ions, which required a wide frequency sweep from $1 \mathrm{MHz}$ to $8 \mathrm{MHz}$, was successfully carried out without resonant frequency tuning.

\section{INTRODUCTION}

The HGC loaded with MA cores has been developed[1] for the JHF (Japan Hadron Facility) proton synchrotrons. The maximum voltage of $20 \mathrm{kV}$ has been obtained by the cavity of $40 \mathrm{~cm}$ in length. The cavity was driven by a pushpull amplifier using two $150 \mathrm{~kW}$ tetrodes. The frequency sweeping from 1 to $5 \mathrm{MHz}$ has been achieved without any tuning circuit because of its broadband characteristics of the MA-loaded cavity[2].

The cavity has been installed in the HIMAC [3] for the beam acceleration to examine the characteristics of the broad-band system. Two major changes has been carried out for both cavity and amplifier. One is to reduce the number of cores to increase the resonant frequency of the cavity. Another is to replace the amplifier. A push-pull amplifier with two $30 \mathrm{~kW}$ tetrodes was newly employed, which covered the required rf frequency range of $1-8 \mathrm{MHz}$ for accelerating heavy ions.

The beam acceleration of $\mathrm{He}, \mathrm{C}, \mathrm{Ne}$ and $\mathrm{Si}$ ions was successfully carried out and the results are reported in this paper. The fundamental $\mathrm{rf}$ and higher harmonics could be driven by the cavity simultaneously because the cavity has a broad impedance, which covers these frequencies. Further improvement of the acceleration efficiency is expected by adding the higher harmonics[4].

Table 1: Parameters of the HGC installed in HIMAC.

\begin{tabular}{ll}
\hline \hline Length & $40 \mathrm{~cm}$ \\
Freq. Range & $1 \sim 8 \mathrm{MHz}$ \\
Gap Voltage & Max. 4 kV \\
Shunt Impedance & $400 \Omega @ 2 \mathrm{MHz}$ \\
& $(4$ cores loaded $)$ \\
Q value & 0.6 \\
Amplifier & $60 \mathrm{~kW}$ push-pull, \\
& $4 \mathrm{CW} 30,000 \mathrm{~A} \times 2$ \\
\hline \hline
\end{tabular}

*E-mail: ryosaku@tanashi.kek.jp

\section{PERFORMANCE OF HGC}

Parameters of the cavity are listed in Table 1.

The cavity loads four MA cores and the total shunt impedance is about $400 \Omega$. Since the MA core has a very low-Q value, the gap voltage can be obtained over a wide range of frequency without tuning system. In this cavity, a half of the peak impedance is guaranteed in the frequency from 1 to $8 \mathrm{MHz}$. The wide-band push-pull amplifier has two tetrodes and each tube is connected to each side of the accelerating gap through the capacitors [5]. Figure 1 shows the cavity impedance measured at the accelerating gap.

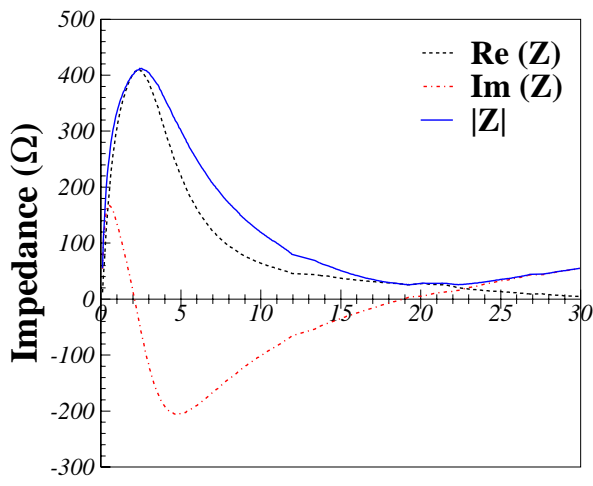

Frequency (MHz)

Figure 1: The measured impedance of HGC.

The maximum gap voltage of $4 \mathrm{kV}$ was achieved at the frequency range from 2 to $3 \mathrm{MHz}$, and the voltage of more than $3 \mathrm{kV}$ was achieved at the frequency range from 1 to $6 \mathrm{MHz}$. The gap voltage of $2 \mathrm{kV}$ was obtained up to the frequency of $8 \mathrm{MHz}$ because the gain of driver amplifier was gradually dropped when the frequency increased.

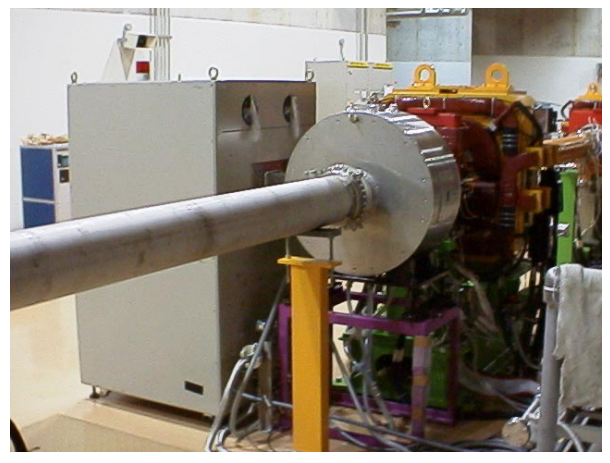

Figure 2: The HGC installed in HIMAC. 
Figure 2 shows the prototype of the HGC installed in HIMAC synchrotron.

\section{EXPERIMENT}

The beam acceleration test was carried out up to the flat top energy of $230 \mathrm{MeV} / \mathrm{u}$ (frequency range: 1.045-5.527 $\mathrm{MHz}$ ) for $\mathrm{He}, \mathrm{C}$ and $\mathrm{Ne}$, and that of $800 \mathrm{MeV} / \mathrm{u}$ for $\mathrm{Si}$ (frequency range: $1.045-7.8 \mathrm{MHz}$ ). Some parameters of the beam are listed in Table 2 .

Table 2: Parameters of the HIMAC synchrotron.

\begin{tabular}{ll}
\hline \hline Injection Energy & $6 \mathrm{MeV} / \mathrm{u}$ \\
Momentum Spread at Injection & $\pm 0.1 \%$ \\
Revolution Frequency at Injection & $261.3 \mathrm{kHz}$ \\
Harmonic Number & 4 \\
Intensity at Injection & $1.0 \times 10^{10} \mathrm{ppp}$ \\
Repetition & $0.3 \mathrm{~Hz}$ \\
Ramping Time & $1 \mathrm{~s}$ \\
\hline \hline
\end{tabular}

Because the particles are injected into the synchrotron as a coasting beam, the adiabatic capture process was employed to increase the capture efficiency. The input signal to the amplifier was adjusted to keep the gap voltage constant because of the frequency dependence of the cavity impedance. Setup of the amplitude modulation of the gap voltage is shown in Fig. 3. In this experiment the modulation signal was programmed beforehand because no feedback loop was necessary.

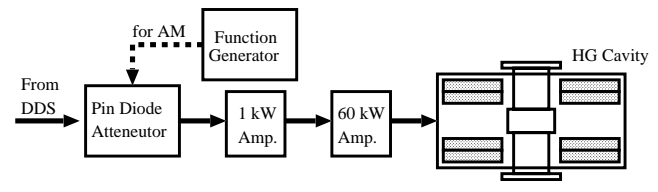

Figure 3: Setup of amplitude modulation.

Figure 4 shows the measured gap voltage. The upper and lower side in Fig. 4 is the input signal and the flattened gap voltage respectively. This shows that the gap voltage of 3 $\mathrm{kV}$ was achieved during whole cycle.

With this system, the beam was adiabatically captured at injection by increasing the rf voltage gradually during 10 $\mathrm{ms}$, which is relatively slow compared with the synchrotron oscillation period of about $1 \mathrm{~ms}$.

The beams of He, $\mathrm{C}$ and $\mathrm{Ne}$ ions were successfully accelerated up to the energy of $230 \mathrm{MeV} / \mathrm{u}$. About $50 \%$ of the beam was accelerated up to the flat top. The beam current was measured by DC Current Transformer (DCCT) as shown in Fig. 5.

For $\mathrm{Si}$ ions, the beam was accelerated up to $800 \mathrm{MeV} / \mathrm{u}$, which was the maximum attainable energy of this ring.

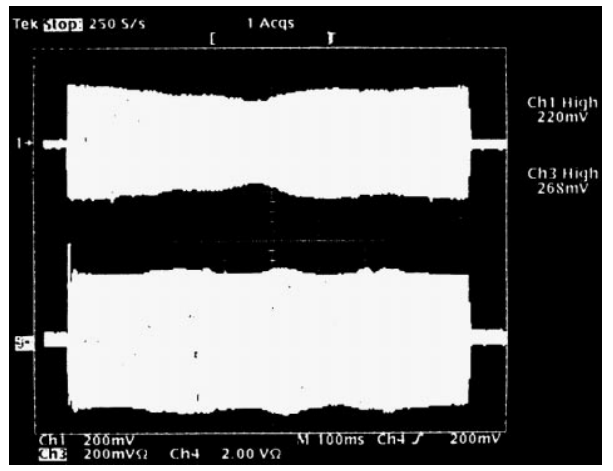

Figure 4: The measured envelope of the gap voltage for $\mathrm{Ne}$ beam acceleration (1.0-5.5 MHz). The rf turned on during $800 \mathrm{~ms}$.

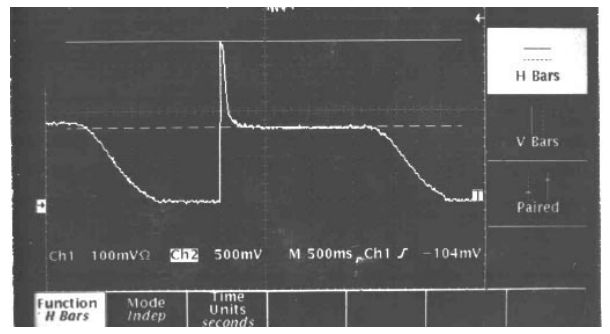

Figure 5: The beam current measured by DCCT. The beam was extracted at the flat top.

\section{SUMMARY}

The first beam acceleration test with HGC was successfully carried out at HIMAC. The accelerating voltage of $3 \mathrm{kV}$ over the frequency range from $1 \mathrm{MHz}$ to $6 \mathrm{MHz}$ was obtained by a small $60 \mathrm{~kW}$ push-pull amplifier without frequency tuning. The beams of $\mathrm{He}, \mathrm{C}, \mathrm{Ne}$ and $\mathrm{Si}$ ions were successfully accelerated with the efficiency of more than $50 \%$.

\section{ACKNOWLEDGMENT}

We would like to thank the members of HIMAC and AEC for operation of HIMAC.

\section{REFERENCES}

[1] Y. Mori et al., 'A New Type of RF Cavity for High Intensity Proton Synchrotron using High Permeability Magnetic Alloy', p.299-p.301, Proc. of EPAC98, 1998.

[2] C. Ohmori et al., 'High Field Gradient Cavity loaded with Magnetic Alloys for Synchrotrons', in this conference.

[3] S. Yamada, 'Commissioning and performance of the HIMAC Medical Accelerator', p.9-p.13, Proc. of PAC95, 1995.

[4] M. Yamamoto et al., 'Multi-Harmonic Acceleration with High Gradient MA Cavity at HIMAC', in this conference.

[5] Y. Sato et al., 'Wide-Band Push-Pull Amplifier for High Gradient Cavity', in this conference. 